\title{
Expressions to implement the IAU 2000 definition of UT1
}

\author{
N. Capitaine ${ }^{1}$, P. T. Wallace ${ }^{2}$, and D. D. McCarthy ${ }^{3}$ \\ 1 Observatoire de Paris, SYRTE/UMR8630-CNRS, 61, avenue de l'Observatoire, 75014 Paris, France \\ ${ }^{2}$ HM Nautical Almanac Office, Space Science and Technology Department, CLRC / Rutherford Appleton Laboratory, UK \\ e-mail: ptw@star.rl.ac.uk \\ 3 US Naval Observatory, 3450 Massachussetts Avenue NW, Washington DC, 20392-5420, USA \\ e-mail: dmc@maia.usno.navy.mil
}

Received 16 January 2003 / Accepted 28 May 2003

\begin{abstract}
This paper provides expressions to be used to implement the new definition of UT1 corresponding to the IAU 2000 resolutions either in the new (CEO-based) or classical (equinox-based) transformations between the International Terrestrial Reference System (ITRS) and the Geocentric Celestial Reference System (GCRS). The new expression for Greenwich Sidereal Time (GST) has to be in agreement at the microarcsecond level, for one century, with the IAU 2000 expressions for the Earth Rotation Angle (ERA) and for the quantity $s$ positioning the Celestial Ephemeris Origin (CEO) on the equator of the CIP. The computations of the new expressions using the IAU 2000 precession-nutation model are performed in such a manner as to ensure that there is no discontinuity in UT1 on 1 January 2003 and that there is equivalence of the classical and new transformations between the ITRS and GCRS relative to the rotation about the axis of the CIP when these expressions are used. The equinox offset that is considered in the computations refers to the dynamical mean equinox of J2000.0. The resulting expressions have been included in the IERS Conventions 2000.
\end{abstract}

Key words. astrometry - reference systems - ephemerides - time

\section{Introduction}

Resolution B1.8 adopted by the IAU in August 2000 recommended that the transformation between the International Terrestrial System (ITRS) and Geocentric Celestial System (GCRS) be specified by the position of the Celestial Intermediate Pole (CIP) in the GCRS, the position of the CIP in the ITRS and the "Earth Rotation Angle" (ERA). This resolution recommended that UT1 be linearly proportional to the ERA, defined as the angle measured along the equator of the CIP between the Celestial Ephemeris Origin (CEO) and the Terrestrial Ephemeris Origin (TEO) through a conventional relationship. This resolution was to be implemented by 1 January 2003, together with Resolution B1.6 adopting the IAU 2000A precession-nutation model for the position of the CIP in the GCRS and Resolution B1.7 for the definition of the CIP. The IERS is moreover to continue to provide users with data and algorithms for the conventional transformations.

Prior to the IAU 2000 definition, UT1 was formally defined by an expression which relates it to mean sidereal time. This expression, which previously was based on Newcomb's expression for the right ascension of the "fictitious mean Sun", was modified for use with the 1976 System of Astronomical Constants, starting on 1984 January 1, 0h UT1. The expression

Send offprint requests to: N. Capitaine, e-mail: capitaine@syrte.obspm.fr for Greenwich Mean Sidereal Time (GMST) at 0h UT1, which was assumed to be the "defining relation" providing the conventional definition of UT1 (Aoki et al. 1982), was developed in order to provide continuity in the value and rate of UT1 as determined from observations at the epoch of change. In realizing this condition, only the effects of changes in position of the catalog equinox from the FK4 to the FK5 and in the precession quantities were considered and the expression was developed to represent a fiducial point with uniform sidereal motion in the new system. The fixed and secular terms in the conventional relationship were fitted to ensure the above conditions, resulting in a change in the "rotational period" of the Earth in UT1, which was considered as a derived quantity.

IAU Resolution B1.8 defines UT1 as linearly proportional to the Earth Rotation Angle (ERA) between the CEO and the TEO, origins on the moving equator which are based on the concept of the "non-rotating origin" (Guinot 1979), in the celestial and terrestrial reference systems respectively. The conventional relationship between ERA and UT1, which has to be considered as being the new defining relation of UT1, does not require modification in order to maintain continuity in UT1 when the models for the motion of the CIP in the GCRS or ITRS are improved or the celestial reference system changed. Therefore, in contrast to the previous approach when the IAU 1976 System was adopted, the implementation of the new definition of UT1 does not require revision of the 
relationship defining UT1 itself, but is based on computation of expressions to be used in the transformation between ITRS and GCRS, once the relationship ERA(UT1) is adopted. This is an important change as compared with the previous procedure. A second change is that this new definition has to be implemented consistently in the new transformation referred to the CEO and in the classical transformation referred to the equinox, which makes it necessary to develop separate expressions for the two options, in each case compliant with the IAU 2000 precession-nutation.

A third change is that, whereas the previous definitions of UT1 were associated with the use of a star catalog and stellar observations, from which UT1 was indirectly derived, the new definition is associated with the use of modern techniques to determine Earth orientation parameters and with the International Celestial Reference System (ICRS) realized by extragalactic radio sources. The new expression for sidereal time, which must now be regarded as a derived expression, has to take into account the equinox offset between the mean equinox at $\mathrm{J} 2000.0$ and the x-origin of the ICRS.

UT1 has been determined observationally essentially from VLBI observations. During the evolution of the VLBI analysis process, it became apparent that there were non-negligible errors in the models for the position of the celestial pole in the celestial reference system. To compensate for these errors, the VLBI analyses were performed solving for "celestial pole offsets". These have been reported by the International Earth Rotation Service (IERS) and were used to establish the MHB2000 nutation model (Mathews et al. 2002), which was adopted as the IAU 2000A model.

The estimates of UT1 determined in this way implicitly include the offset in longitude and the correction to the 1976 precession in longitude in the equation of the equinoxes. Also, recall that in 1997 the expression for Greenwich Sidereal Time (GST) was amended with the addition of two complementary terms to make the definition consistent with observational accuracy. As a result, the observational values of UT1 reported by the IERS do not strictly refer to the conventional IAU definition of UT1 (Aoki et al. 1982). In fact, since the IAU 2000A model closely approximates the observed celestial pole offsets, the values of UT1 reported until this time have been consistent with the IAU 2000A precession-nutation. Implementation of the IAU resolutions on 1 January 2003 requires that a new definition of UT1 be determined that is in agreement with past observational practices as well as the IAU resolutions.

The purpose of this paper is to provide expressions to implement the new definition of UT1 based on these IAU 2000 resolutions, either in the new (CEO-based) or classical (equinox-based) transformations between the ITRS and GCRS. The new expression for Greenwich Sidereal Time, GST, has to be in agreement at the microarcsecond level, for one century, with the conventional relationship between the Earth Rotation Angle (ERA) and UT1 and with the IAU 2000 expressions for the quantity $s$ positioning the CEO on the equator of the CIP. The computations of the new expressions using the IAU 2000 precession-nutation model (Mathews et al. 2002) are performed in such a manner as to ensure that there is no discontinuity in UT1 on 1 January 2003 and that there is equivalence of the classical and new transformations between the ITRS and GCRS relative to the rotation about the axis of the CIP when these expressions are used. This requires us to determine the corresponding conventional values to be adopted in these expressions for the parameters which are sensitive to the models and have a critical role in ensuring continuity.

The paper first summarizes in Sect. 2 the necessary considerations for the computations and gives in Sects. 3 and 4 the details of the computation of the expressions for $s$ and GST. Then, Sect. 5 presents the tests for equivalence between the two transformations, Sect. 6 reports on the comparison between the current and new procedures and Sect. 7 provides the numerical expressions which fulfill the above requirements. The expression for GST is provided as the sum of the two following expressions:

* (41) for Greenwich Mean Sidereal Time;

* (44) for the "Equation of the Equinoxes", expressed as the sum of the classical part and "complementary terms",

and the definitive expression (46) for the polynomial part of the quantity $s$ locates the CEO in a consistent way with the above expressions. Additionally, an electronic table, available at http://maia.usno.navy.mil/ch5tables.html, provides the full numerical expression for GST00, including its polynomial part (41) for GMST and the definitive and full form of the complementary terms to be added to the classical equation of the equinoxes in (44). Finally, Sect. 8 provides the practical way to implement the new definition of UT1 according to the procedure used and Sect. 9 summarizes the main points of the paper.

\section{Considerations for the computation of the Earth Rotation Angle and Sidereal Time}

The Earth Rotation Angle (ERA), $\theta$, is obtained by the use of its conventional relationship with UT1 as given by Capitaine et al. (2000),

$$
\begin{aligned}
\theta\left(T_{\mathrm{u}}\right)= & 2 \pi(0.7790572732640 \\
& \left.+1.00273781191135448 T_{\mathrm{u}}\right),
\end{aligned}
$$

where

$T_{\mathrm{u}}=($ Julian UT1 date -2451545.0$)$,

and

$$
\begin{aligned}
\mathrm{UT} 1= & \mathrm{UTC}(\text { Coordinated Universal Time }) \\
& +(\mathrm{UT} 1-\mathrm{UTC}) .
\end{aligned}
$$

The parameter $t$, used in the following for the development of the various expressions, is defined by:

$t=(\mathrm{TT}-2000$ January $1 \mathrm{~d} 12 \mathrm{~h} \mathrm{TT}) / 36525$.

with TT in days.

As mentioned in the Introduction, there are two equivalent ways of implementing Resolution B1.8 in the transformation from ITRS to GCRS, namely (a) the new paradigm based on 
the direct use of the CEO and the ERA and (b) the classical paradigm based on the equinox and GST, but using the CEO and the ERA indirectly. They are called respectively "CEObased" and "equinox-based" transformations in the following.

Although neither the ITRS nor the GCRS involves the equinox or the CEO directly, the classical and new transformations do use these distinct longitude zero-points during the course of the transformation. This raises questions of nomenclature, in particular what to call the equivalent in the new system of the classical right ascension. It can be argued that because historically right ascensions have always been with respect to an equinox it is improper to use the same term for the CEO-based case. By analogy with the well-established use of the terms right ascension and declination in specifying ICRS coordinates, we take the pragmatic view that the introduction of the CEO as an alternative zero-point does not justify a change in terminology. Thus we use the term right ascension in a generic way, meaning the longitude coordinate of any Earthpole-based celestial coordinate system; it, may be referred to the CEO (or the ICRS prime meridian) as well as some sort of equinox.

In our convention, the $\mathrm{CEO}$ is thus the origin of right ascension of date in the new system, the counterpart of true equinox of date in the classical system. The two origins of course have quite different properties. The equinox is defined geometrically and has a complex and comparatively rapid motion along the instantaneous equator that is is a consequence of the motion not only of the moving equator but of the moving ecliptic as well. The CEO, which is an implementation of the non-rotating origin (Guinot 1979), in contrast is defined kinematically: from one moment to the next, it moves only at right-angles to the instantaneous equator, and no ecliptic is involved.

This almost complete separation between the treatment of the precessing-nutating pole and the origin of right ascension leads to a much simpler relationship between stellar hour angles and Universal Time. Note also that the CEO is at present almost stationary in ICRS right ascension, remaining within 0.1 arcsec of the ICRS prime meridian for the whole of the 21 st century and beyond.

The CEO-based transformation uses the conventional relationship between the ERA and UT1 together with the quantity $s(t)$ providing the position of the CEO, $\sigma(\equiv \sigma(t))$, on the equator of the CIP. This quantity is defined kinematically, and depends on an integration that uses the history of the CIP coordinates $(X, Y)$, as given by the IAU 2000 A precession-nutation model. The quantity $s$ corresponding to a finite displacement of the CIP between epochs $t_{0}$ and $t$ is such that:

$s=\sigma N-\Sigma_{0} N-\left(\sigma_{0} N_{0}-\Sigma_{0} N_{0}\right)$,

where $\sigma_{0}=\sigma\left(t_{0}\right), \Sigma_{0}$ is the origin on the equator of the GCRS, and $N_{0}$ and $N$ are the ascending nodes of the equators at $t_{0}$ and $t$ in the equator of the GCRS. The quantity $\sigma_{0} N_{0}-\Sigma_{0} N_{0}$ is an arbitrary constant, which will be denoted $C_{0}$ in the following.

The equinox-based transformation uses a conventional relationship between GMST and UT1 together with an expression for the "equation of the equinoxes" providing the difference between Greenwich (apparent) Sidereal Time, GST, and GMST. The kinematical definition of GST refers to the kinematical meaning of the equation of the equinoxes (see Sect. 4.4), which refers the Earth rotation to the CEO. This is defined as the sum of two parts, the classical part and the complementary terms. For further details see Aoki \& Kinoshita (1983) and Capitaine $\&$ Gontier (1993). It will be called in the following the "complete equation of the equinoxes". These expressions have to be derived in conjunction with precession and nutation quantities derived from the IAU 2000A precession-nutation model.

This equinox-based transformation has to take into account the frame bias about the $z$-axis, $\mathrm{d} \alpha_{0}$, which is the right ascension of the mean equinox at epoch, $\gamma_{0}$, in the GCRS. This component of the frame bias is the arc such that $\mathrm{d} \alpha_{0}=\Sigma_{0} N_{0}-\gamma_{0} N_{0}$, where $\Sigma_{0}$ is the $x$-origin of the GCRS, $\gamma_{0}$ is the mean equinox of $\mathbf{J} 2000$, and $N_{0}$ is the node between the mean equator at $\mathbf{J} 2000$ and the equator of the GCRS. Note that this $\mathrm{d} \alpha_{0}$ rotation does not cause shifts in the right ascensions of stars in either the GCRS or the intermediate frame. It merely re-orients the underlying classical precession-nutation model, eliminating a small but spurious distortion that would otherwise be present. The corresponding shifts in the "true" right ascensions are canceled by compensating changes to the new sidereal time formulas. The numerical value, $\mathrm{d} \alpha_{0}=-14.6$ mas, used for this equinox offset is that provided by Chapront et al. (2002) which corresponds to the inertial definition of the equinox. See Appendix A and Capitaine et al. (2003) for further discussion.

The numerical development used for positioning the CEO on the equator of the CIP is for $s+X Y / 2$ rather than $s$ itself, because the former requires fewer terms to reach a given accuracy (Capitaine 1990). There is, moreover, a helpful similarity between the quantity $s+X Y / 2$, which equals, up to the 3rd order in $X$ and $Y$, the GCRS right ascension of the CEO, and the complementary terms in the complete equation of the equinoxes, which represent the right ascension of the CEO in the mean equatorial frame at $\mathrm{J} 2000$. In the expression for $s+X Y / 2$ (cf. Eq. (7)), the amplitudes of the trigonometric terms are due to cross terms between precession and nutation and between the biases and the nutations. The coefficients of the polynomial part, except the secular term, originate from cross terms between the coefficients of the polynomial parts of $X$ and $Y$. The secular term contains both the cross term between the bias in $Y$ and the precession rate in $X$ and the effect of the nutations. For more detail, see Sect. 4.4.

Given the above considerations, the following conditions have to be fulfilled to ensure consistency between the z-rotation component of the CEO-based and equinox-based transformation between GCRS and ITRS:

(i) the relationship between GMST and UT1 has to be based on the conventional relationship (1) between the ERA and UT1;

(ii) the secular term in the expressions for $s$ and GST have to be such that the contribution from the coupling between nutations and between precession and frame biases (see expressions (21) and (23)) is the same in the final zrotations;

(iii) the arbitrary position, $\sigma_{0}$, of the CEO at J2000 on the true equator at $\mathrm{J} 2000$ has to be chosen to be consistent with the frame bias about the $z$-axis, $\mathrm{d} \alpha_{0}$; 
(iv) the conventional value of $s$ at epoch (J2000) has to be chosen so that it is consistent both with the position, $\sigma_{0}$, of the CEO at J2000 and with the convention for the value at epoch of the complementary terms in the equation of the equinoxes.

Furthermore, to ensure the continuity of UT1 at the date of implementation of the IAU Resolutions, the following conditions must be met:

(v) no discontinuity in UT1 between old and new systems on 1 Jan. 2003;

(vi) no change in old and new directions in the GCRS on 1 Jan. 2003;

(vii) no change in old and new terrestrial coordinates on 1 Jan. 2003.

The unavoidable discontinuity in UT1 rate at the date of change will be discussed in Sects. 3.4 and 7.

We also note the following:

(1) The conventional relationship (1) between $\theta$ and UT1 is related to the current relationship between GMST and UT1 so that, if $\theta_{0}$ and $\theta_{1}$ are the fixed and secular terms respectively (see Capitaine et al. 1986):

$\theta_{0}=\theta(\mathbf{J} 2000)=\operatorname{GMST}(\mathbf{J} 2000)$,

$\theta_{1}=\left(\mathrm{GMST}_{1}\right)_{1982}-A-A_{\mathrm{s}}$, with $A$ being the speed of precession in right ascension based on the IAU 1976 precession and $A_{\mathrm{s}}$ the coefficient of the secular term in the quantity $s$ due to the IAU 1980 nutation.

(2) A consequence of measures taken in the past to keep UT1 continuous whenever improvements in the GST(UT1) relation were introduced is that GMST and the equation of the equinoxes no longer mean, geometrically, "the GHA of the mean equinox" and "the true RA of the mean equinox", thus compromising any strictly classical interpretation. The changes on 1 January 2003 carry this process one stage further.

(3) The two largest terms that have already been introduced in the "equation of the equinoxes" (cf. IAU Resolution C7, Recom. 3, 1994), on 1 Jan. 1997 when they were close to zero, were really to make ERA(UT1) correspond better to the Earth rotation angle with respect to an inertial reference system, and were not in principle changes either to GMST or the equation of the equinoxes in a geometrical sense; the equation of the equinoxes currently in use, will be called in the following "the IAU 1994 equation of the equinoxes", $E E_{1994}$.

(4) The value of the complementary terms introduced into the equation of the equinoxes on 1 Jan. 1997 was not exactly equal to zero at that date, but to $-134 \mu \mathrm{as}$, and its value at J2000.0 was $+2102 \mu$ as (see Table 2).

(5) We must expect the provisional convention $s(0)=0$ to be at odds with other constraints, in particular $\mathrm{d} \alpha_{0}$ and $\theta_{0}$.

(6) A consequence of the current VLBI estimations of UT1 which do not take into account any equinox offset is that, in practice, GMST(J2000) actually refers to the GCRS origin of right ascension and not to the mean equinox of epoch, as it is generally understood.

(7) The current VLBI estimations of UT1 make use of the "celestial pole offsets" to correct the equation of the equinoxes (see Sect. 6), which has the consequence of deriving UT1 from a "practical" relationship between GMST and UT1 which differs from the conventional relationship by correcting the discrepancies in the precession-nutation model in ecliptic longitude.

(8) The values of the constant and secular terms both in $s$ and GST are the critical conventional values to be chosen to ensure that there is (i) equivalence between the CEO and equinoxbased transformations for the estimation of UT1 and UT1 rate and (ii) that there is no jump in UT1 and the least possible discontinuity in dUT1/dt on 1 Jan. 2003.

The above statements have to be taken into account when determining the constant value at J2000.0 of both $s$ and GST, as well as the secular term in their expressions. The adoption of numerical values for these terms is the final step in providing the conventional expressions for these quantities (see Sect. 7.2).

\section{Computation of the quantity $s$}

\subsection{Semi-analytic expression}

The expression for $s$ as a function of the coordinates $X$ and $Y$ is (Capitaine et al. 2000)

$s=-\int_{t_{0}}^{t} \frac{(X \dot{Y}-Y \dot{X})}{1+Z} \mathrm{~d} t-C_{0}$,

the conventions for $s(0)$ and $C_{0}$ being discussed in Sect. 3.3.

The above formulation allows one to predict $s$ directly as a function of time. Note that applications requiring $s$ could, in principle, perform a numerical integration to evaluate expression (6). However, such approaches would usually be too computationally expensive to be practical, hence the need for an expression as a function of time.

A semi-analytical expression including a polynomial of $t$ and a trigonometric part depending on the literal fundamental arguments of nutation was computed with the GREGOIRE software package, designed to manipulate Poisson series. The computation used IAU-2000A-based expressions for the coordinates $X$ and $Y$ as functions of time, relations (20) and (21) in Capitaine et al. (2000) and produced an expression for $s$ including all the terms larger than $0.1 \mu$ as (Capitaine et al. 2003) in the form:

$$
\begin{aligned}
s(t)= & -X Y / 2+\sum_{i=0} s_{i} t^{i} \\
& +\sum_{j=0} \sum_{k}\left[\left(C_{s, j}\right)_{k} \sin \alpha_{k}+\left(C_{c, j}\right)_{k} \cos \alpha_{k}\right] t^{j} .
\end{aligned}
$$

In the provisional model, the constant term for $s$ was chosen so that $s(J 2000)=0$.

A limitation of the semi-analytical computation is due to the introduction, through the integration process, of very small divisors associated with critical combination of arguments which produce a "numerical noise" without any physical 
Table 1. Comparison between the expression for $s+X Y / 2-X(0) Y(0) / 2$ as a function of time and a pure numerical integration using the pole trajectory, unit: $\mu$ as.

\begin{tabular}{ccc}
\hline \hline Provisional model & Fitted value & Degree \\
\hline 2184 & 2184.7 & constant \\
+3812 & +3808.35 & $t$ \\
-121.15 & -119.94 & $t^{2}$ \\
-72574.09 & -72574.09 & $t^{3}$ \\
+27.70 & +27.69 & $t^{4}$ \\
+15.61 & +15.65 & $t^{5}$ \\
\hline
\end{tabular}

meaning, not acceptable to achieve a given precision. The computation was therefore limited to periods lower than 500 years and the contribution to $s$ from nutation terms with periods greater than 500 years has been considered as a contribution to the secular and quadratic terms for which the notation $s_{\mathrm{LPN}}$ will be used. This contribution has been evaluated by the use of a rigorous numerical integration as described below.

\subsection{Rigorous numerical integration}

The alternative method, which we used for our computations, is to implement the CEO's kinematical definition literally, by a numerical integration of Capitaine et al. (1986) expression (2.2):

$s=\int_{t_{0}}^{t}(\cos d-1) \dot{E} \mathrm{~d} t+C_{0}$.

Almost identical results were obtained by numerically integrating the alternative expression (6) using rectangular coordinates.

We used this direct numerical integration procedure to compute $s$ over an interval from 1800 to 2200, with time steps typically of 0.1 day but as small as 0.005 day in some trials. These predictions were compared with the $s$ expression (provisional model) by taking differences every 200 days and fitting a polynomial in $t$. The results are shown in Table 1. Prior to fitting, the residuals stay within a few microarcseconds from 1900 to 2100 . The contribution $s_{\text {LPN }}$ has been evaluated (in $\mu$ as) to be $+16 t+1 t^{2}$.

The small change to the fixed term is meaningless, because the integration was initialized in agreement with the model, and the other adjustments are not significant. The final fitted value for the constant term will be provided in Sect. 7.2.

This numerical integration, which has confirmed the semianalytical expression for $s$ as a function of time at the level of a few microarcseconds after one century, has provided the definitive expression for the secular and quadratic terms in the expression.

\subsection{Convention to be adopted for the position of the CEO at J2000 on the mean equator of $\mathrm{J} 2000$}

The expression for the quantity $s$ provides the position of the $\mathrm{CEO}$ on the equator of the CIP, given the arbitrary constant, $C_{0}$ which is related to $\sigma_{0}$, the position of the CEO at $\mathbf{J} 2000$ on
Table 2. Values (in $\mu$ as) of the periodic component in $s+X Y / 2$ and in the complementary terms in the equation of the equinoxes (IAU 1994 and IAU 2000 expressions) at different epochs.

\begin{tabular}{cccc}
\hline \hline Epoch & $s+X Y / 2$ & $E E_{c_{1994}}$ & $E E_{c_{2000}}$ \\
\hline $01 / 01 / 1997$ & +124 & -134.0 & -123.5 \\
2000.0 & -2107 & +2102.1 & +2107.2 \\
$01 / 01 / 2003$ & +2476 & -2476.0 & -2476.4 \\
\hline
\end{tabular}

the equator of the CIP at J2000 (see relation (5)). This position has to be chosen in order to ensure consistency between the CEO-based and the equinox-based transformations, taking into account the frame bias about the $z$-axis, $\mathrm{d} \alpha_{0}$.

\subsubsection{Simplified hypothetical case}

In order to understand more easily the role of this $\mathrm{d} \alpha_{0}$ rotation in both transformations, let us assume that the biases in $X$ and $Y$ are zero and that nutation is zero. In the equinox-based transformation, then, the $\mathrm{d} \alpha_{0}$ rotation would transform the GCRS, through the rotation matrix $\boldsymbol{B}$ (see Eq. (30)), to the mean equatorial frame at $\mathbf{J} 2000$. The precession quantities would then transform the mean equatorial frame of J2000.0 to the equatorial frame of date linked to the CIP and equinox of date.

In the CEO-based transformation for this simplified case, the GCRS would be transformed to the intermediate frame of date linked to the CIP and CEO, using the quantities $E$ and $d$ which include both the effect of the $z$-bias and the precession. This would transform to a frame linked to the CIP and to an origin $\Sigma$ on the equator of the CIP, such that $\Sigma N$ would equal $\Sigma_{0} N$. Then $s$ would be used to define the CEO kinematically.

The arbitrary constant $C_{0}$ related to the position of the CEO at $\mathrm{J} 2000$, could therefore be chosen such that $\sigma_{0} N_{0}$ would equal $\Sigma_{0} N_{0}$, which means that $\sigma_{0}$ is chosen in order that the rotation to the equinox at epoch annuls $\mathrm{d} \alpha_{0}$.

\subsubsection{Constant term in the expressions for $s$}

The constant terms in the formulas for $s$ and $s+X Y / 2$ have in the past been chosen in order that $s(\mathrm{~J} 2000)$ would equal 0 , which means that:

$$
\begin{aligned}
s(0)= & \frac{1}{2} X(0) Y(0) \\
& -\sum_{k}\left[\left(C_{s, 0}\right)_{k} \sin \alpha_{k}(0)+\left(C_{c, 0}\right)_{k} \cos \alpha_{k}(0)\right] .
\end{aligned}
$$

However, the constraints mentioned in Sect. 2 lead to a different value, due in particular to the following:

(i) the distance at epoch between the CEO and the equinox (see Sect. 3.3.1),

(ii) the conventional relationship between $\theta$ and UT1 such that, at J2000, $\theta=$ GMST, which would theoretically have the effect, in essence, of placing the CEO at the position of the IAU 1976 J2000 mean equinox,

(iii) continuity in UT1 with the value corresponding to the current relationship on 1 Jan. 2003, including the contribution 
of the IAU 2000 correction to the precession rate from epoch to that date.

The value of the fixed term in $s+X Y / 2$ as provided in Table 1 has now been re-evaluated in order to fulfill the above conditions. See Sect. 7.2.

\subsection{Secular term in the expression for $s$}

Consistent values for (i) the secular term in $s$ and (ii) the secular nutation contribution in GMST should be adopted in order that the continuity of UT1 rate be ensured in the best possible way when changing from the current expression of GST to either the new expression for GST or the expression for $s, \theta(U T 1)$ being a conventional relationship.

Concerning the secular term in the expression for $s$, it has been noted (see Sect. 2) that the IAU 2000 relationship (1) has been derived from the 1982 conventional expression for GMST(UT1) in order that the secular contribution of the current conventional model for nutation be included in GMST. Note that this contribution represents the secular term in the corresponding expression for $s$. The numerical value used in this computation was $+3850 \mu \mathrm{as} / \mathrm{c}$, the value consistent with the IAU 1980 nutation and no offsets of the CIP at J2000. The updated value for the secular term, $s_{\mathrm{sec}}$, in $s$, consistent with the IAU 2000 precession-nutation and its associated offsets at $\mathbf{J} 2000$, is given by:

$s_{\mathrm{sec}}=(3856+16-33) \mu \mathrm{as} / \mathrm{c}=3839 \mu \mathrm{as} / \mathrm{c}$

which is the sum of the contributions from the IAU $2000 \mathrm{nu}-$ tations with periods smaller than 500 years (first term), and periods greater than 500 years (second term) and from the IAU 2000 frame bias in obliquity (third term).

Continuity in UT1 rate, between the current GMST(UT1) relationship and the new one based on the CEO, would require that the secular contribution from nutation in the expressions for $s$ corresponding to the old and new model be the same, whereas there is an unavoidable difference of $11 \mu \mathrm{as} / \mathrm{c}$ due to the improvements in the model.

Moreover, it should be noted that the definition of the Earth Rotation Angle requires the use of a quantity $s^{\prime}$ for positioning the TEO in the ITRS, which is at present modeled as $s^{\prime}=-47 \mu$ as $t$ (see Lambert \& Bizouard 2002). This secular amount adds to that of $-s$ in the new expression.

This shows that continuity in UT1 rate cannot be strictly ensured when using expressions consistent with the IAU 2000A precession-nutation model.

\section{Computation of Greenwich Sidereal Time}

\subsection{Relation to Earth Rotation Angle}

The IAU 2000 expression for GST has to be consistent with (i) the IAU 2000A precession-nutation, (ii) the expression (1) for $\theta(\mathrm{UT} 1)$, and (iii) the expression for $s$ based on the IAU $2000 \mathrm{~A}$ precession-nutation.

The computation of this expression is based on the following relation to the Earth Rotation Angle, retaining all the terms needed to achieve a final accuracy of one microarcsecond:

$$
\begin{aligned}
\mathrm{GST}= & \mathrm{d} T_{0}+\theta+\widehat{\widehat{\gamma_{0} \sigma_{0}}} \\
& +\int_{0}^{t}\left(\psi_{\mathrm{A}} \hat{\dot{+\Delta}} \psi_{1}\right) \cos \left(\omega_{\mathrm{A}}+\Delta \epsilon_{1}\right) \mathrm{d} t-\chi_{\mathrm{A}} \\
& +\Delta \psi \cos \epsilon_{\mathrm{A}}-\Delta \psi_{1} \cos \omega_{\mathrm{A}},
\end{aligned}
$$

where $\psi_{\mathrm{A}}, \omega_{\mathrm{A}}$ and $\epsilon_{\mathrm{A}}$ are the precession quantities corrected for the IAU 2000 precession rates; $\Delta \psi_{1}$ and $\Delta \epsilon_{1}$ are the nutation quantities referred to the ecliptic of epoch which can be derived from the IAU 2000A series for the nutation quantities $\Delta \psi$ and $\Delta \epsilon$ referred to the ecliptic of date. $\chi_{\mathrm{A}}$ is the planetary precession along the equator.

The second and third lines in (11) account for the accumulated precession and nutation in right ascension from J2000 to the epoch $t$. The difference GST $-\theta$ provides the right ascension of the CEO measured from the equinox along the moving equator and $\mathrm{d} T_{0}$ is a constant term to be fitted in order to ensure continuity in UT1 at the date of change (1 January 2003).

The computation has been performed in a semi-analytical form using the same software for products, derivatives and integration of polynomials and trigonometric series as that used for the computation of the quantity $s$. The resulting development is a polynomial in $t$, which, by convention, will be considered as being GMST, plus the classical "equation of the equinoxes", $\Delta \psi \cos \epsilon_{\mathrm{A}}$, plus a series of periodic terms similar to those appearing in the quantity $s+X Y / 2$ (with nearly the same amplitudes and with opposite sign). The following expression is consistent with those for $\theta$ and $s$ :

$$
\begin{aligned}
\mathrm{GST}= & \mathrm{d} T_{0}+\theta+4612^{\prime \prime} 15739966 t \\
& +1^{\prime \prime} 39667721 t^{2} \\
& -0{ }^{\prime \prime} 00009344 t^{3}+0{ }^{\prime \prime} 00001882 t^{4} \\
& +\Delta \psi \cos \epsilon_{\mathrm{A}} \\
& +\sum_{k}\left[\left(C_{s, 0}^{\prime}\right)_{k} \sin \alpha_{k}+\left(C_{c, 0}^{\prime}\right)_{k} \cos \alpha_{k}\right] \\
& -00^{\prime \prime} 00000087 t \sin \Omega .
\end{aligned}
$$

Note that the polynomial part of (12) (i.e. the first three lines of the expression) provides the IAU 2000 expression for Greenwich Mean Sidereal Time, GMST, whereas the periodic part provides the complete "equation of the equinoxes" of which the non-classical part (also called the "complementary terms") replaces the two complementary terms of the IAU 1994 equation of the equinoxes provided in the IERS Conventions 1996 (McCarthy 1996).

The IAU 2000A expression for the complementary terms of the equation of the equinoxes, is provided by Table 3 and is also included in the electronic table providing the full numerical expression for GST, available at http://maia.usno.navy.mil/ch5tables.html

A secular term similar to that appearing in the quantity $s$ is included in the expression for GMST and the contribution from nutation terms with very long periods (greater than 500 years) is taken into account in a form similar to the contribution $-s_{\mathrm{LPN}}$ in the quantity $-s$ (see Sect. 3.2). For more detail on the constant and secular term of this expression see Sects. 4.5 and 4.6. 
Table 3. Terms with amplitudes $\geq 0.1 \mu$ as in the IAU 2000A expression for the complementary terms of the equation of the equinoxes. $l, l^{\prime}, F, D, \Omega$ are the Delaunay variables and $L_{\mathrm{Ve}}, L_{\mathrm{E}}$ the mean longitudes of Venus and the Earth respectively. The coefficients are in $\mu$ as.

\begin{tabular}{|c|c|c|}
\hline Argument $\alpha_{k}$ & $\left(C_{s, 0}^{\prime}\right)_{k}$ & $\left(C_{c, 0}^{\prime}\right)_{k}$ \\
\hline$\Omega$ & +2640.96 & -0.39 \\
\hline $2 \Omega$ & +63.52 & -0.02 \\
\hline $2 F-2 D+3 \Omega$ & +11.75 & +0.01 \\
\hline $2 F-2 D+\Omega$ & +11.21 & +0.01 \\
\hline $2 F-2 D+2 \Omega$ & -4.55 & +0.00 \\
\hline $2 F+3 \Omega$ & +2.02 & 0.00 \\
\hline $2 F+\Omega$ & +1.98 & 0.00 \\
\hline $3 \Omega$ & -1.72 & 0.00 \\
\hline$l^{\prime}+\Omega$ & -1.41 & -0.01 \\
\hline$l^{\prime}-\Omega$ & -1.26 & -0.01 \\
\hline$l+\Omega$ & -0.63 & 0.00 \\
\hline$l-\Omega$ & -0.63 & 0.00 \\
\hline$l^{\prime}+2 F-2 D+3 \Omega$ & 0.46 & 0.00 \\
\hline$l^{\prime}+2 F-2 D+\Omega$ & 0.45 & 0.00 \\
\hline $4 F-4 D+4 \Omega$ & 0.36 & 0.00 \\
\hline$F-D+\Omega-8 L_{\mathrm{Ve}}+12 L_{\mathrm{E}}$ & -0.24 & -0.12 \\
\hline $2 F$ & 0.32 & 0.00 \\
\hline $2 F+2 \Omega$ & 0.28 & 0.00 \\
\hline$l+2 F+3 \Omega$ & 0.27 & 0.00 \\
\hline$l+2 F+\Omega$ & 0.26 & 0.00 \\
\hline $2 F-2 \Omega$ & -0.21 & 0.00 \\
\hline$l^{\prime}-2 F+2 D-3 \Omega$ & 0.19 & 0.00 \\
\hline$l^{\prime}-2 F+2 D-\Omega$ & 0.18 & 0.00 \\
\hline $8 L_{\mathrm{Ve}}-13 L_{\mathrm{E}}$ & -0.10 & 0.05 \\
\hline $2 D$ & 0.15 & 0.00 \\
\hline $2 l-2 F-\Omega$ & -0.14 & 0.00 \\
\hline$l-2 D+\Omega$ & 0.14 & 0.00 \\
\hline$l^{\prime}+2 F-2 D+2 \Omega$ & -0.14 & 0.00 \\
\hline$l-2 D-\Omega$ & 0.14 & 0.00 \\
\hline $4 F-2 D+4 \Omega$ & 0.13 & 0.00 \\
\hline $2 F-2 D+4 \Omega$ & -0.11 & 0.00 \\
\hline$l-2 F-3 \Omega$ & 0.11 & 0.00 \\
\hline$l-2 F-\Omega$ & 0.11 & 0.00 \\
\hline$\Omega$ & $-0.87 \times t$ & 0.00 \\
\hline
\end{tabular}

\subsection{Correction $d G M S T$ to the current relationship between GMST and UT1}

The IAU 2000 expression for GMST can also be expressed as the sum of the current expression for GMST(UT1) and the difference dGMST between the polynomial part of the IAU 2000 expression for GST and the current GMST expression. The new expression for GST can then be provided by the sum of the new expression for dGMST plus the new expression for the equation of the equinoxes.
This method is equivalent to the previous one except for the time scale on which the computation of the accumulated precession in right ascension (i.e. UT in the 1982 GMST relationship and TT in the new GST) is based. More detail on the effect of the time scales on the GST expression is given in Sect. 4.3.

The numerical expression for dGMST has thus been derived from the polynomial part of the expression (12) for GST, minus the difference between $\mathrm{GMST}_{1982}$ (UT1) and $\theta(\mathrm{UT1} 1)$. In $\mu$ as, it is:

$$
\begin{aligned}
\mathrm{dGMST}= & \mathrm{d} T_{0}+\left(\mathrm{d} T^{\prime}-\mathrm{d} T_{0}^{\prime}\right)-274950.12 t+117.21 t^{2} \\
& -0.44 t^{3}+18.82 t^{4},
\end{aligned}
$$

where $\mathrm{d} T^{\prime}$ and $\mathrm{d} T_{0}^{\prime}$ are the contributions from the difference (TT - UT1) at the date $t$ and the epoch J2000 respectively.

\subsection{Time scales used in the expression for GST}

The new expression for GST as a function of time clearly distinguishes between $\theta$, which is expressed as a function of UT1, and the accumulated precession-nutation in right ascension. This part, being derived from the precession-nutation quantities, which are expressed in TDB (or, in practice, TT), is consequently expressed in TT.

This is not the case in the $\mathrm{GMST}_{1982}$ (UT1) expression which used only UT1 and, therefore, mixes the two time scales UT1 and TT (see Capitaine et al. 1986). This inconsistency had no significant consequence at the time (1982) when the GMST(UT1) relationship was adopted. However, at the start of 2003, TT - UT1 $\simeq 64.4$ s, which, when multiplied by the speed of precession in right ascension, gives differences of about $94 \mu$ as in the GST prediction and therefore in the estimated UT1 (see Gontier 2002). More precisely, using TT - TAI = $32.184 \mathrm{~s}$, the effect can be expressed as:

$\mathrm{d} T^{\prime}=47 \mu$ as $+1.46 \mu$ as $(\mathrm{TAI}-\mathrm{UT} 1)$

where TAI - UT1 is in seconds.

This is an example of the benefits of the clear separation between ERA and precession-nutation in the new CEO-based transformation. It also shows that the estimation of UT1 based on the current relationship GMST(UT1), includes a systematic error of the order $100 \mu$ as due to the incorrect use of UT for computing precession in RA in GST. This effect has also to be considered when ensuring continuity of UT1 on 1 Jan. 2003.

\subsection{Relation to the quantity $s$}

Expression (11) can be written as:

$\mathrm{GST}=\theta+\widehat{\gamma \sigma}$.

It is the sum of the ERA and the accumulated precession and nutation along the moving equator, reckoned from the CEO, from epoch to the date $t$. It can also be written as the sum of a polynomial part, GMST, and a periodic part, the "complete equation of the equinoxes" (i.e. in a kinematical sense, referred to the CEO: see Sect. 2). 
The contribution to GST from precession is:

$\mathrm{dGST}_{\text {prec }}=\int_{0}^{t} \dot{\psi_{\mathrm{A}}} \cos \omega_{\mathrm{A}} \mathrm{d} t-\chi_{\mathrm{A}}$,

or, retaining all the terms to ensure an accuracy of one microarcsecond for a century:

$$
\begin{aligned}
\mathrm{dGST}_{\mathrm{prec}}= & \psi_{\mathrm{A}} \cos \epsilon_{0}-\chi_{\mathrm{A}} \\
& -\int_{0}^{t} \dot{\psi_{\mathrm{A}}} \sin \epsilon_{0}\left(\omega_{\mathrm{A}}-\epsilon_{0}\right) \mathrm{d} t
\end{aligned}
$$

where $\epsilon_{0}$ is the mean obliquity at epoch.

The contribution to GST from nutation and coupling between precession and nutation can be written as:

$E E_{\mathrm{dyn}}=E E_{\mathrm{cl}}+\mathrm{d} E E_{\mathrm{s}}+\mathrm{d} E E_{\mathrm{p}}$,

the expression for the classical part of the equation of the equinoxes being:

$E E_{\mathrm{cl}}=\Delta \psi \cos \epsilon_{\mathrm{A}}$,

where $\epsilon_{\mathrm{A}}$ is the mean obliquity of date.

At the microarcsecond level, we can write (see Aoki \& Kinoshita 1983 and Capitaine \& Gontier 1993):

$$
\begin{aligned}
\mathrm{d} E E_{\mathrm{p}}= & -\int_{0}^{t} \dot{\psi_{\mathrm{A}}} \sin \omega_{\mathrm{A}} \Delta \epsilon_{1} \mathrm{~d} t \\
& -\int_{0}^{t}\left[\dot{\overrightarrow{\Delta \psi_{1}}} \Delta \epsilon_{1} \sin \omega_{\mathrm{A}} \mathrm{d} t\right]_{\mathrm{p}} \\
& -\frac{1}{2}\left[\int_{0}^{t} \dot{\psi_{\mathrm{A}}} \Delta \epsilon_{1}^{2} \cos \omega_{\mathrm{A}} \mathrm{d} t\right]_{\mathrm{p}}, \\
\mathrm{d} E E_{\mathrm{s}}= & -\left[\int_{0}^{t} \dot{\overrightarrow{\Delta \psi_{1}}} \Delta \epsilon_{1} \sin \omega_{\mathrm{A}} \mathrm{d} t\right]_{\mathrm{s}} \\
& -\frac{1}{2}\left[\int_{0}^{t} \dot{\psi_{\mathrm{A}}} \Delta \epsilon_{1}^{2} \cos \omega_{\mathrm{A}} \mathrm{d} t\right]_{\mathrm{s}},
\end{aligned}
$$

using the same notations as in (11) and the subscripts "p" and "s" being for the periodic and secular components of the expressions, respectively.

The relation between the quantity $s$ and the complete equation of the equinoxes can be derived by equating the rotation along the equator of the CIP from the node $N$ (see Sect. 2) to the TEO in the two transformations.

This provides, at the microarcsecond level:

$$
\begin{aligned}
s= & +\frac{1}{2} \eta_{0} \psi_{\mathrm{A}} \sin \epsilon_{0}-\mathrm{d} E E_{\mathrm{s}} \\
& +\int_{0}^{t} \dot{\psi_{\mathrm{A}}} \sin \omega_{\mathrm{A}}\left(\omega_{\mathrm{A}}-\epsilon_{0}\right) \mathrm{d} t-\frac{1}{2} \psi_{\mathrm{A}} \sin \epsilon_{0}\left(\omega_{\mathrm{A}}-\epsilon_{0}\right) \\
& +\frac{1}{12} \psi_{\mathrm{A}}^{3} \sin ^{2} \epsilon_{0} \cos \epsilon_{0} \\
& -\mathrm{d} E E_{\mathrm{p}}-\frac{1}{2} \Delta \psi_{1} \Delta \epsilon_{1} \sin \epsilon_{0} \\
& -\frac{1}{2} \psi_{\mathrm{A}} \sin \epsilon_{0} \Delta \epsilon_{1}+\frac{1}{2}\left(\omega_{\mathrm{A}}-\epsilon_{0}\right) \sin \epsilon_{0} \Delta \psi_{1} \\
& +\frac{1}{4} \sin ^{2} \epsilon_{0} \cos \epsilon_{0}\left(\psi_{\mathrm{A}}^{2} \Delta \psi_{1}+\psi_{\mathrm{A}} \Delta \psi_{1}^{2}\right) \\
& +\frac{1}{12}\left(\psi_{\mathrm{A}}\right)^{3} \sin \epsilon_{0} \Delta \epsilon_{1}
\end{aligned}
$$

The two first lines in (22) are the polynomial components, including the secular component, $s_{\mathrm{S}}$ (first line), or:

$s_{\mathrm{s}}=+\frac{1}{2} \eta_{0} \psi_{\mathrm{A}} \sin \epsilon_{0}-\mathrm{d} E E_{\mathrm{s}}$

and the precession (second line), which can also be written as:

$$
\begin{aligned}
s_{\text {prec }}= & \sum_{i=2} s_{i} t^{i}=\frac{1}{2} \sin \epsilon_{0} \int_{0}^{t}\left[\dot{\psi_{\mathrm{A}}}\left(\omega_{\mathrm{A}}-\epsilon_{0}\right)-\dot{\omega_{\mathrm{A}}} \psi_{\mathrm{A}}\right] \mathrm{d} t \\
& +\frac{1}{12} \sin ^{2} \epsilon_{0} \cos \epsilon_{0} \psi_{\mathrm{A}}^{3} .
\end{aligned}
$$

The third line in (22) is the periodic component,

$$
\begin{aligned}
s_{\mathrm{p}} & =\sum_{k}\left[\left(C_{s, 0}\right)_{k} \sin \alpha_{k}+\left(C_{c, 0}\right)_{k} \cos \alpha_{k}\right] \\
& =-\mathrm{d} E E_{\mathrm{p}}-\frac{1}{2} \Delta \psi_{1} \Delta \epsilon_{1} \sin \epsilon_{0},
\end{aligned}
$$

and the fourth and fifth lines are the Poisson terms,

$$
\begin{aligned}
s_{t^{n} \times \mathrm{per}}= & +\sum_{j=0} \sum_{k}\left[\left(C_{s, j}\right)_{k} \sin \alpha_{k}+\left(C_{c, j}\right)_{k} \cos \alpha_{k}\right] t^{j} \\
= & -\frac{1}{2} \psi_{\mathrm{A}} \sin \epsilon_{0} \Delta \epsilon_{1}+\frac{1}{2}\left(\omega_{\mathrm{A}}-\epsilon_{0}\right) \sin \epsilon_{0} \Delta \psi_{1} \\
& +\frac{1}{4} \sin ^{2} \epsilon_{0} \cos \epsilon_{0}\left(\psi_{\mathrm{A}}^{2} \Delta \psi_{1}+\psi_{\mathrm{A}} \Delta \psi_{1}^{2}\right) \\
& +\frac{1}{12}\left(\psi_{\mathrm{A}}\right)^{3} \sin \epsilon_{0} \Delta \epsilon_{1} .
\end{aligned}
$$

The above expression (22) (or more specifically (24), (25) and (26)) thus provides the relation between $s$ and the complementary terms $\mathrm{d} E E_{\mathrm{s}}$ and $\mathrm{d} E E_{\mathrm{p}}$ in the complete equation of the equinoxes, which will be used in the following sections.

\subsection{Constant term in the expressions for the complementary part of the equation of the equinoxes}

In the provisional model for the complementary terms in the equation of the equinoxes, the constant term has been chosen so that it compensates the value of its periodic component at $\mathrm{J} 2000$. Its numerical value, which is mainly due to its two first terms, is provided by Table 2 at different dates of importance for the IERS Conventions.

Note that the two first periodic terms of $\sum_{k} C_{k}^{\prime} \sin \alpha_{k}$ have already been included in the expression of GST since 1 Jan. 1997 as complementary terms to the classical equation of the equinoxes (see Sect. 2). Note also that the constant term of the final $z$-rotation in the equinox-based transformation is the value at epoch (see expression (25)) of the quantity $\mathrm{d} E E_{\mathrm{p}}+\frac{1}{2} \Delta \psi_{1} \Delta \epsilon_{1} \sin \epsilon_{0} \simeq \mathrm{d} E E_{\mathrm{p}}+\frac{1}{2} X Y$.

The constant contribution of these terms is now considered as being part of GMST.

\subsection{Secular term in the complementary terms of the equation of the equinoxes}

The secular term in GST contains one term, originating from the computation of the "complete equation of the equinoxes", 
which is a contribution from nutation, similar to that appearing in $s$ (see 3.4). We elect to include this secular component in the formula for GMST. Note that the secular term of the final $z$-rotation from the node in the equinox-based transformation (see expression (23)) includes the contributions both from nutation $\left(\mathrm{d} E E_{\mathrm{s}}\right)$ and frame bias $\left(-\frac{1}{2} \eta_{0} \psi_{\mathrm{A}} \sin \epsilon_{0}\right)$. Concerning the requirement for ensuring continuity in UT1 rate, see Sect. 3.4.

\section{Comparison between new and classical transformation}

\subsection{Description of the method}

We have compared the new (CEO-based) and classical (equinox-based) transformations from GCRS to ITRS, with polar motion omitted. The comparisons were made through the GCRS-to-ITRS rotation matrix, which we call $\boldsymbol{R}$, with elements $\boldsymbol{R}(i, j)$.

The new form of the transformation is written, using our usual notation, as:

$\boldsymbol{R}_{\text {new }}=R_{3}(\theta-E-s) \cdot R_{2}(d) \cdot R_{3}(E)$,

where $\theta$ is the Earth Rotation Angle provided by relation (1), and

$E=\arctan (Y / X)$,

$d=\arctan \left(\left(\left(X^{2}+Y^{2}\right) /\left(1-X^{2}-Y^{2}\right)\right)^{1 / 2}\right)$.

$X(t)$ and $Y(t)$ are the coordinates of the CIP unit vector in the GCRS, based on the IAU 2000A precession-nutation model and the corresponding biases $\left(\xi_{0}, \eta_{0}\right)$ and $\mathrm{d} \alpha_{0}$ is the equinox offset at epoch.

For this comparison the quantity $s(t)$ is that derived from expression (6) using the CIP coordinates $(X, Y)$, corresponding to the IAU 2000A precession-nutation model, as provided by Capitaine et al. (2003), with the final polynomial part as computed in Sect. 3, with the working assumption that $s$ equals zero at epoch (J2000), (i.e. the constant term in $s$ being equal to $2184 \mu \mathrm{as})$.

The classical form of the transformation is written as

$$
\boldsymbol{R}_{\text {class }}=\boldsymbol{T N P B}
$$

or as the product of the individual rotation matrices $\boldsymbol{B}$ (bias) followed by $\boldsymbol{P}$ (precession) then $\boldsymbol{N}$ (nutation) and finally $\boldsymbol{T}$ (Earth rotation):

$$
\begin{aligned}
\boldsymbol{B} & =R_{1}\left(-\eta_{0}\right) \cdot R_{2}\left(\xi_{0}\right) \cdot R_{3}\left(\mathrm{~d} \alpha_{0}\right), \\
\boldsymbol{P} & =R_{3}\left(\chi_{\mathrm{A}}\right) \cdot R_{1}\left(-\omega_{\mathrm{A}}\right) \cdot R_{3}\left(-\psi_{\mathrm{A}}\right) \cdot R_{1}\left(\epsilon_{0}\right), \\
\boldsymbol{N} & =R_{1}\left(-\left[\epsilon_{\mathrm{A}}+\Delta \epsilon\right]\right) \cdot R_{3}(-\Delta \psi) \cdot R_{1}\left(\epsilon_{\mathrm{A}}\right) \\
\boldsymbol{T} & =R_{3}(\mathrm{GST}) .
\end{aligned}
$$

The classical quantities $\psi_{\mathrm{A}}, \omega_{\mathrm{A}}$ and $\epsilon_{\mathrm{A}}$ are from Lieske et al. (1977) plus IAU 2000A corrections to the precession rates, and the nutation quantities $\Delta \psi$ and $\Delta \epsilon$ are the luni-solar and planetary nutations from the IAU 2000A series. GST is Greenwich (apparent) Sidereal time, derived from either expression for GST or dGMST provided in Sects. 4.1 and 4.2 respectively.
For this comparison, the constant term in the expression for GMST is $-2107 \mu$ as, chosen to make the contribution of the complementary terms in the equation of the equinoxes equal zero at epoch (J2000) (see Sect. 4.5).

Note that the precession matrix, $\boldsymbol{P}$, can be formed in several ways (Williams 1994), depending on which of the Lieske et al. (1977) angles is used. The four-angle formulation given above has the advantage that the IAU 2000A precession-rate adjustments can be applied directly and unambiguously. The threeangle formulation using $\zeta_{\mathrm{A}}, \theta_{\mathrm{A}}$ and $z_{\mathrm{A}}$ is more common, but in this case applying the IAU $2000 \mathrm{~A}$ precession-rate adjustments is not as straightforward. Note also that $\zeta_{\mathrm{A}}, \theta_{\mathrm{A}}$ and $z_{\mathrm{A}}$ polynomials are not suitable for combining frame bias and precession in one step, because of difficulties close to epoch. Other threeangle polynomial formulations are possible that overcome this difficulty.

\subsection{Consistency checks relating to the position of the CIP}

For both the classical and new transformations, elements $(3,1)$ and $(3,2)$ of the $\boldsymbol{R}$ matrix are the coordinates $X, Y$ of the CIP in the GCRS. Accordingly, our comparisons of the classical and new CIP predictions consisted simply of comparing $X$ and $Y$ obtained directly (see Capitaine et al. 2000) with elements $(3,1)$ and $(3,2)$ of $\boldsymbol{R}_{\text {class. }}$.

When we carried out the classical transformations for $\boldsymbol{B}, \boldsymbol{P}$ and $\boldsymbol{N}$ as above, namely as three separate, rigorous, steps (for more detail see Wallace 2002), we achieved an agreement of a few $\mu$ as between the classical and new methods. However, if we omitted the bias, used the Lieske et al. (1977) precession model without adjustments, and then used the IAU 2000A "total" nutations (i.e. the nutations themselves plus the contribution of the corrections to the precession rates plus the biases), errors reaching 1 mas after a century were seen. Furthermore, differences of a similar size were seen if different choices of precession angles were made, because of limitations set by the Lieske et al. (1977) series. More details concerning these tests are given in Capitaine et al. (2003).

\subsection{Consistency checks for the rotation about the z-axis}

We have used three methods of forming the GCRS-to-ITRS matrix for the purpose of computing the $z$-rotation difference:

(I) $\boldsymbol{R}_{\text {new }}$ based on the $X, Y, \theta$ and $s$ expressions,

(II) $\boldsymbol{R}_{\text {class }}$, with GST $=$ expression (12), with $\theta$ as in (1), and

(III) $\boldsymbol{R}_{\text {class }}$ with GST $=$ GMST $_{1982}+$ dGMST + complete equation of the equinoxes, with dGMST $=$ expression (13).

The difference in rotation about the $z$-axis (i.e. the axis of the CIP) between the two transformation matrices $R_{\text {new }}$ and $R_{\text {class }}$ has been derived by the following procedure. When two rotation matrices $\boldsymbol{A}$ and $\boldsymbol{B}$ differ only by a rotation $\phi$ about the final $z$-axis, we can write:

$\boldsymbol{B}=R_{3}(\phi) \cdot \boldsymbol{A}$. 


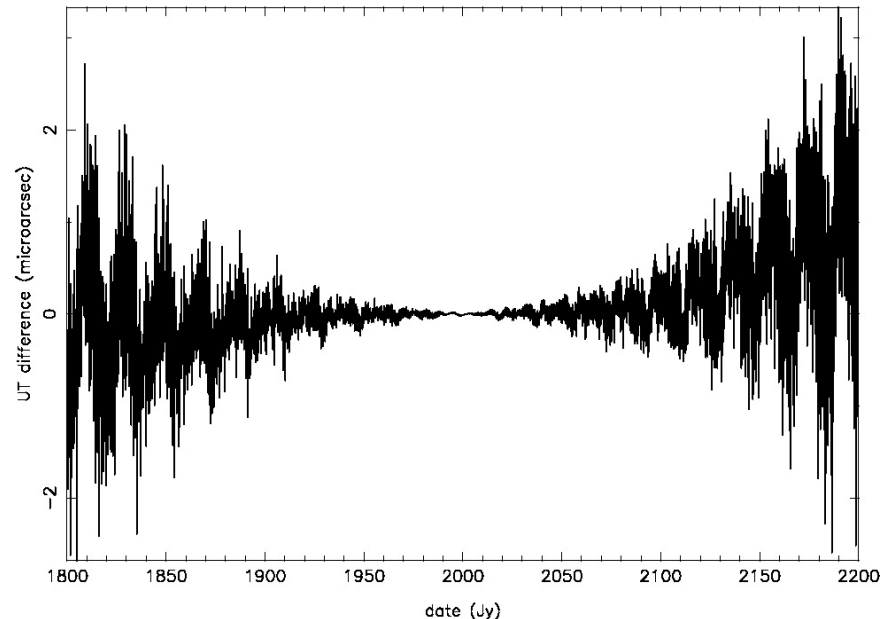

Fig. 1. $z$-rotation difference, expressed as UT difference, between the equinox-based and CEO-based transformations over 2 centuries around $\mathbf{J} 2000$.

To solve for $\phi$, we take the product of each side with the inverse (=transpose) of the first matrix:

$\boldsymbol{B} \cdot \boldsymbol{A}^{\mathrm{T}}=R_{3}(\phi) \cdot \boldsymbol{A} \cdot \boldsymbol{A}^{\mathrm{T}}$

or:

$\boldsymbol{B} \cdot \boldsymbol{A}^{\mathrm{T}}=R_{3}(\phi)$.

Writing the left-hand side as $\boldsymbol{D}$ and expanding the $R_{3}$ rotation, we have:

$\boldsymbol{D}=\boldsymbol{B} \cdot \boldsymbol{A}^{\mathrm{T}}=\left(\begin{array}{ccc}\cos \phi & \sin \phi & 0 \\ -\sin \phi & \cos \phi & 0 \\ 0 & 0 & 1\end{array}\right)$

and hence the angle $\phi$ is given by

$\phi=\arctan \frac{(\boldsymbol{D}(1,2)-\boldsymbol{D}(2,1))}{(\boldsymbol{D}(1,1)+\boldsymbol{D}(2,2))}$.

(Four elements have been used rather than two in order to produce an average value for $\phi$ in the presence of rounding errors or a rotation which is not purely about $z$.)

The comparison has been performed over several centuries around $\mathbf{J} 2000$. The $z$-rotation differences obtained in this comparison are very similar for (I) versus (II) and (I) versus (III).

We note that, in order that the $z$-rotations be equivalent in the CEO-based and equinox-based transformation, the constant term to be used in $s$ is minus the value at epoch of the quantity $s+X Y / 2$ (see Table 2), instead of that of $s$ (see Table 1). Once the constant term is removed, the agreement is of the order of a few $\mu$ as over the four centuries between the two transformations (see Fig. 1 for the plot of the corresponding UT difference).

This comparison shows that the rotation about the CIP axis is the same at the microarcsecond level in the CEO-based and equinox-based transformations when using, in the CEO-based transformation, the semi-analytical expressions for the coordinates of the CIP in the GCRS and for the quantity $s$ based on the IAU 2000 precession-nutation model (Capitaine et al. 2003, and Sect. 3) together with the IAU 2000 expression of
$\theta(\mathrm{UT} 1)$ and, in the equinox-based transformation, the classical precession and nutation quantities and frame biases based on the IAU 2000 precession-nutation model together with the semi-analytical IAU 2000 expression (12) for GST.

\section{Comparison between new and current estimation of UT1}

The current estimation of UT1 from observations makes use of the "IERS (VLBI) celestial pole offsets" to correct the equation of the equinoxes in using the term $\mathrm{d} \psi \cos \epsilon_{\mathrm{A}}$, where $\mathrm{d} \psi$ is the celestial offset in ecliptic longitude, including the correction to the IAU 1976 precession model, the IAU 1980 nutation model and the frame bias $\mathrm{d} \psi_{0}$ at $\mathrm{J} 2000$. Such a method actually derives UT1 from a value of GST which corresponds to:

(i) the GMST(1982) expression (Aoki et al. 1982) corrected for the error in the IAU 1976 precession in longitude;

(ii) the correction to the equation of the equinoxes based on the IAU 1976 value for the mean obliquity of date, $\epsilon_{\mathrm{A}}$;

(iii) the equation of the equinoxes corrected for the errors in the amplitudes of the IAU 1980 nutation in longitude;

(iv) the equation of the equinoxes corrected for the FCN contribution;

(v) the term $\mathrm{d} \psi_{0} \cos \epsilon_{\mathrm{A}}$ (i.e. $39510 \mu$ as using the IERS 1996 offset at epoch and $38327 \mu$ as using the IAU 2000 value).

This is equivalent (at the order of accuracy of the MHB model) to deriving UT1 from a new expression for GST using the IAU 2000A precession-nutation model and the associated biases, plus the FCN effects, but including only the effect of bias and precession rate in $\psi_{\mathrm{A}}$ and not in $\epsilon_{\mathrm{A}}$.

Note that the current VLBI procedure does not use the rigorous transformation as described in the previous section, but uses a procedure which considers the precession and frame biases corrections as if they were nutations. This introduces spurious secular and quadratic terms in the estimated UT1 and also cancels the contribution $\left(\xi_{0} \cot \epsilon_{0}\right)$ from the frame bias in longitude (cf. point (v) above) to GMST.

Figure 2 shows UT differences between the current VLBI procedure and the rigorous (or CEO-based) method with the final fitted values for the constant terms (see the following section). Figure 3 shows the same differences but with the complementary contribution of a modeled variation of TT - UT1. These Figures show that, in the long term, the differences are dominated by a quadratic term; this comes from the correction to the precession rate in obliquity which was not corrected in the current VLBI procedure, whereas the main secular difference is due to the variation in TT - UT1. The periodic differences originate from the new terms considered in the expression of the complementary terms in the equation of the equinoxes.

The above considerations together with those already noted in Sect. 2 are important points to take into account for ensuring continuity in UT1 on 1 Jan. 2003. In order to be consistent with the current reduction of observations, the correct procedure would be not to ensure continuity with the current expression for GMST(UT1) based on the IAU 1976 precession and the IAU 1994 equation of the equinoxes. It would rather 


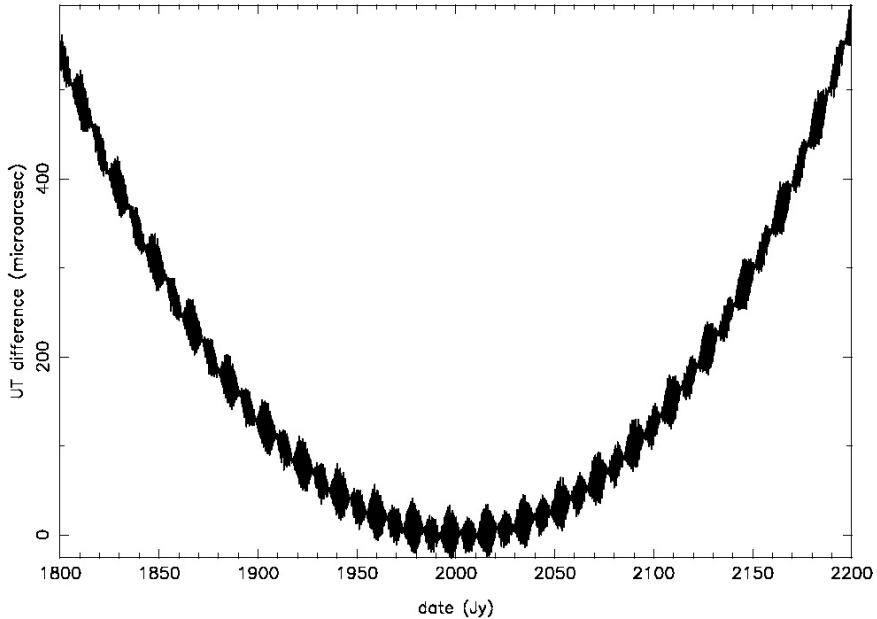

Fig. 2. UT differences between the current VLBI reduction (with fixed TT - UT1) and CEO-based over 2 centuries around J2000.

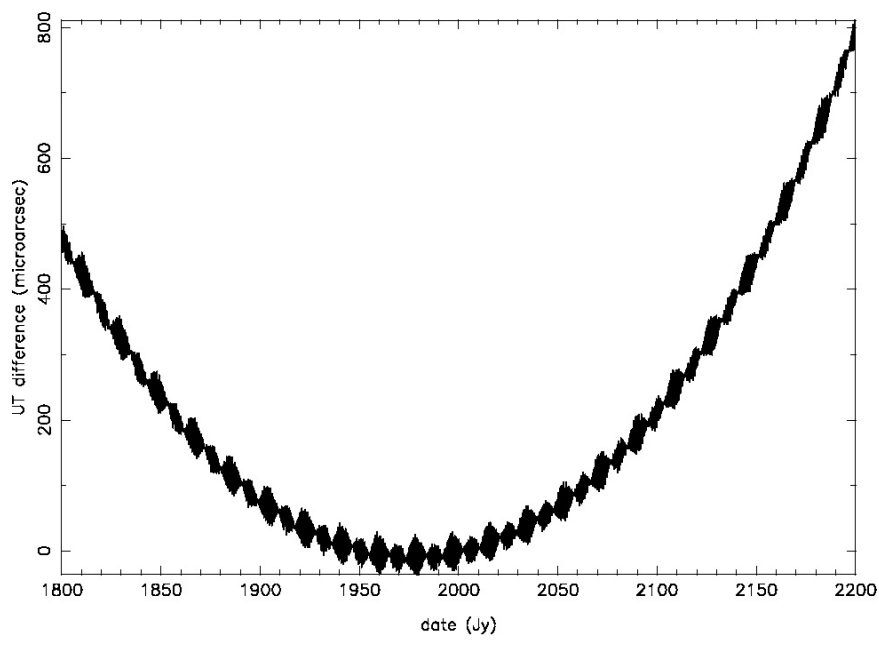

Fig. 3. UT differences between the current VLBI reduction and CEObased over 2 centuries around J2000, with an approximate model for TT - UT1.

be to ensure continuity with an expression for GST which is already corrected for a part of the imperfection in the current precession-nutation model, using the correction to the equation of the equinoxes based on the estimated celestial pole offset.

Moreover, it is also necessary to take into account that the future estimations of UT1 will continue to use the correction $\mathrm{d} \psi \cos \epsilon_{\mathrm{A}}$ to the equation of the equinoxes derived from the observed celestial pole offsets $\mathrm{d} \psi$, which will be corrections to the IAU 2000A precession-nutation model.

\section{Expressions to implement the IAU 2000 definition of UT1}

\subsection{Conditions to ensure the continuity of UT1}

IAU Resolution B1.8 recommends that the definition of UT1 be provided by the relationship (1) between the ERA and UT1

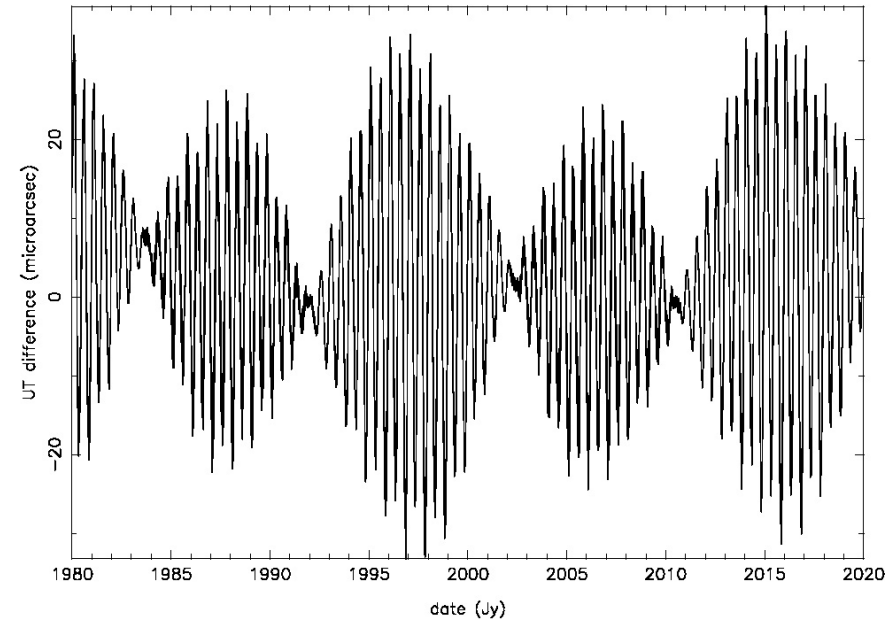

Fig. 4. UT differences between the current VLBI reduction and CEObased, with fixed TT - UT1 over 40 years around J2000.

which ensures that the time derivative of UT1 is proportional to the Earth's angular velocity $\omega$.

The continuity in UT1 which has to be ensured on 2003 Jan. 1.0 TT (i.e. JD 2452640.5 TT) requires that, at that date, the rotation about the axis of the CIP is the same when using the current VLBI procedure to estimate UT1 and the new procedure based on the expressions developed in the previous sections to implement the IAU 2000 definition of UT1.

As already mentioned, continuity in UT1 rate cannot be strictly ensured between the old and new procedures, due to the use of both a conventional ERA(UT1) relationship and new models of which aim is to provide an ERA derivative which represents more accurately the Earth's angular velocity.

The discontinuity in UT1 rate will be due to (i) improvement in the computation of the secular contribution of nutation (see Sects. 3.4 and 4.6), (ii) the use of the new quantity $s^{\prime}$ for positioning the TEO, (iii) the introduction of additional terms in the expression for the dynamical equation of the equinoxes (and equivalently for $s$ ), (iv) the distinction between TT and UT1 in the new model for GST, (v) the improvement in the precession rate in obliquity.

Figure 4 shows the UT differences over 40 years around J2000 between the current estimation and the new estimation based on the new models. This shows that the differences at present are dominated by the incompleteness of the IAU 1994 expression for the equation of the equinoxes.

The two constraints at play in order (a) to ensure the equivalence between the CEO-based and equinox-based transformations for providing UT1 and (b) to ensure the continuity of UT1 when changing from the old to the new models for precession, nutation and GST are

(a) The $z$-rotation component of the GCRS-to-ITRS matrix must be the same in both the CEO-based and equinoxbased formulations at any date.

(b) At 2003 Jan. 1.0, the $z$-rotation component must be the same using the old and new procedures. 
Note that this is a generalization of the condition which was used in Aoki et al. (1982), but restricted at that time to precession and equinox bias.

Constraint (a) has been shown to be verified (cf. Sect. 5.3) when using expression (12) for GST as a function of $\theta$, expression (7) for $s$ and the same constant term in the expressions for GMST and $s$. However, the only condition imposed by constraint (a) for the constant term, given the relationship for $\theta$ (UT1), is that it must be the same in the two expressions, the value of this term having to be determined by the numerical equivalence required by constraint (b) on 1 January 2003.

In order that constraint (b) be verified, the relation to be used is

$R_{z}\left(\boldsymbol{R}_{\text {class }}\right)_{\text {old }}=R_{z}\left(\boldsymbol{R}_{\text {class }}\right)_{\text {new }}$

the $z$-rotation being based on the old and new expression for GST respectively and on the old and new precession and nutation quantities provided by the old and new models respectively, and the relation between GST and UT1 being consistent with relation (1) between $\theta$ and UT1.

The expression for $\mathrm{GST}_{\text {old }}$ is such that, at 2003 Jan. 1.0 TT,

$\mathrm{GST}_{\mathrm{old}}=\mathrm{GMST}_{1982}(\mathrm{UT} 1)+E E_{\mathrm{IERS} 1996}$,

where $E E_{\mathrm{IERS} 1996}$ is the equation of the equinoxes recommended in the IERS 1996 Conventions:

$E E_{\mathrm{IERS} 1996}=E E_{1994}+\mathrm{d} \psi \cos \epsilon_{\mathrm{A}}$,

$E E_{1994}$ being (see Sect. 2 (3)), the IAU 1994 equation of the equinoxes:

$E E_{1994}=\Delta \psi \cos \epsilon_{\mathrm{A}}+\mathrm{d} E_{1}+\mathrm{d} E_{2}$,

$\mathrm{d} E_{1}$ and $\mathrm{d} E_{2}$ being the two complementary terms added in the IAU 1994 expression.

The models for computing the quantities in the old system are thus based on:

(i) the current relationship between GMST and UT1;

(ii) the IAU 1976 precession;

(iii) the IAU 1980 nutation;

(iv) the IAU 1994 expression for the two complementary terms, $\mathrm{d} E_{1}$ and $\mathrm{d} E_{2}$ in the equation of the equinoxes.

The check for the equivalence in $z$-rotation has to consider that the pre-2003 VLBI analysis procedure includes the time dependent celestial pole offsets as additions to the quantities provided by the old precession-nutation model, as recommended in the IERS Conventions. The use of the IAU 2000 precessionnutation model together with that of the corresponding correction to the equation of the equinoxes is a similar procedure. The models for computing the quantities in the new system are based on:

(i) the IAU 2000 precession and nutation;

(ii) expression (12) for $\mathrm{GST}_{\text {new }}$.

\subsection{Determination of the IAU 2000 expressions linking GST and ERA and locating the CEO}

The objective is to derive values for the terms in GMST and $s+X Y / 2$ so that the various methods of computing the rotational orientation of the Earth - old GST/equinox, new GST/equinox, and ERA/CEO - all agree at 2003 Jan. 1.0 TT = JD 2452640.5 . In order to achieve this agreement we need to predict TAI - UT1 for that date, and a value of $32.3 \mathrm{~s}$ was adopted, corresponding to TT $-\mathrm{UT} 1=64.484 \mathrm{~s}$. Given the TT $(\simeq$ TDB $)$ and UT1 dates, and neglecting both polar motion and the free core nutation, we are in a position to calculate the constant term in GMST. Given that term, the CEO can be located by making a suitable choice of fixed term in the $s+X Y / 2$ expression. The procedure used was as follows:

1. Use the IAU $1980 \mathrm{~J} 2000$ obliquity, $\epsilon_{0}$.

2. Compute the interval $t$ between the fundamental epoch J2000.0 and the TT transition date, in Julian centuries.

3. Obtain the IAU 1980 obliquity $\epsilon_{\mathrm{A}}$ for date $t$.

4. Compute the Lieske et al. (1977) precession angles, for date $t,\left(\psi_{\mathrm{A}}\right)_{1977},\left(\omega_{\mathrm{A}}\right)_{1977}$ and $\chi_{\mathrm{A}}$.

5. Evaluate the IAU $2000 \mathrm{~A}$ nutation components $\Delta \psi$ and $\Delta \epsilon$ for date $t$. The IAU 2000A model provides separate components for the frame bias $\left(\Delta \psi_{\mathrm{bi}}\right.$ and $\left.\Delta \epsilon_{\mathrm{bi}}\right)$, the precession rate adjustments $\left(\Delta \psi_{\mathrm{pr}}\right.$ and $\left.\Delta \epsilon_{\mathrm{pr}}\right)$ and the nutation proper (luni-solar $\Delta \psi_{\mathrm{ls}}$ and $\Delta \epsilon_{\mathrm{ls}}$ ) and (planetary $\Delta \psi_{\mathrm{pl}}$ and $\Delta \epsilon_{\mathrm{pl}}$ ).

6. Using expression (1), calculate the Earth Rotation Angle, $\theta$, as a function of UT1.

7. Use expression (7) to compute for date $t$ the quantity $s$ and the periodic terms required by expression (12). Note that the value of the fixed term in $s+X Y / 2$ is immaterial as we are about to calculate the correction to apply. Also, estimate the quantity $s^{\prime}$, from $s^{\prime}=-47 t$ (in microarcseconds).

8. Compute the IAU $1976\left(\zeta_{\mathrm{A}}, z_{\mathrm{A}}, \theta_{\mathrm{A}}\right)$ precession matrix for date $t$.

9. Using $\left(\epsilon_{\mathrm{A}}\right)_{1977}$, apply the IAU 2000A "total" nutations $\Delta \psi_{\mathrm{bi}+\mathrm{pr}+\mathrm{ls}+\mathrm{pl}}$ and $\Delta \epsilon_{\mathrm{pr}+\mathrm{pr}+\mathrm{ls}+\mathrm{pl}}$ to the matrix: see the third line of expression (30). The matrix now contains the effects of frame bias, corrected precession, and nutation.

10. Compute the IAU 1982 GMST, using UT1 throughout, giving $\mathrm{GMST}_{\text {old }}$.

11. Using $\Delta \psi_{\mathrm{bi}+\mathrm{pr}+\mathrm{ls}+\mathrm{pl}}$, evaluate the IAU 1994 equation of the equinoxes $E E_{1994}$ for date $t$.

12. Form $_{\mathrm{GST}_{\text {old }}}=\mathrm{GMST}_{\text {old }}+E E_{1994}$.

13. Use $\mathrm{GST}_{\text {old }}$ to apply a $z$-rotation to the matrix, which becomes $\left(\boldsymbol{R}_{\text {class }}\right)_{\text {old }}$. Note that the quantity $s^{\prime}$ is currently neglected.

14. Apply the IAU 2000A precession corrections by computing $\psi_{\mathrm{A}}=\left(\psi_{\mathrm{A}}\right)_{1977}+\Delta \psi_{\mathrm{pr}}, \omega_{\mathrm{A}}=\left(\omega_{\mathrm{A}}\right)_{1977}+\Delta \epsilon_{\mathrm{pr}}$ and $\epsilon_{\mathrm{A}}=$ $\left(\epsilon_{\mathrm{A}}\right)_{1980}+\Delta \epsilon_{\mathrm{pr}}$.

15. Use the first line of expression (30) to compute the frame bias matrix; $\mathrm{d} \alpha_{0}=-14.6$ mas, $\xi_{0}=\Delta \psi_{\text {bi }} \sin \epsilon_{0}$ and $\eta_{0}=\Delta \epsilon_{0}$.

16. Use $\epsilon_{0}, \psi_{\mathrm{A}}, \omega_{\mathrm{A}}$ and $\chi_{\mathrm{A}}$ and the second line of expression (30) to apply the (corrected) precession to the matrix.

17. Use $\epsilon_{\mathrm{A}}$ and the "pure" nutations $\Delta \psi_{\mathrm{ls}+\mathrm{pl}}$ and $\Delta \epsilon_{\mathrm{ls}+\mathrm{pl}}$ and the second line of expression (30) to apply the nutation to the matrix. 
18. Use the first part of expression (12) to compute the Greenwich Mean Sidereal Time GMST ${ }_{\text {new }}$. Note that the UT1 has already been used to calculate the ERA $\theta$ and the time argument for the remainder of the expression is the TT date $t$. Note also that the value of the constant term is immaterial as we are about to calculate the correction to apply.

19. Use the second part of expression (12) to compute, for date $t$, the new equation of the equinoxes $E E_{\text {new }}$, using the corrected obliquity $\epsilon_{\mathrm{A}}$ and the "pure" nutation in longitude $\Delta \psi_{\mathrm{ls}+\mathrm{pl}}$.

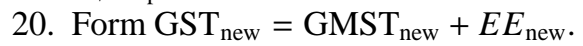

21. Use GST $T_{\text {new }}$ plus the quantity $s^{\prime}$ to apply a $z$-rotation to the matrix, which becomes $\left(\boldsymbol{R}_{\text {class }}\right)_{\text {new }}$.

22. Using 5.3, determine the $z$-rotation difference between $\left(\boldsymbol{R}_{\text {class }}\right)_{\text {old }}$ and $\left(\boldsymbol{R}_{\text {class }}\right)_{\text {new }}$. This is the correction to whatever fixed term was used when calculating $\mathrm{GMST}_{\text {new }}$, earlier, and is the first result.

23. Apply the correction to the current value of GMST new $_{\text {. }}$

24 . Use the correction also to complete $\left(\boldsymbol{R}_{\text {class }}\right)_{\text {new }}$ by applying it as a $z$-rotation.

25. Extract $X$ and $Y$ from the matrix $\left(\boldsymbol{R}_{\text {class }}\right)_{\text {new }}$; these are the coordinates of the CIP at date $t$.

26. Use $X$ and $Y$ in expression (28) to obtain the angles $E$ and $d$.

27. Use $X, Y, s$ and $\theta$ to construct the new, CEO-based, GCRSto-ITRS matrix $\boldsymbol{R}_{\text {new }}$ by means of expression (27).

28. Using 5.3, determine the $z$-rotation difference between $\boldsymbol{R}_{\text {new }}$ and $\left(\boldsymbol{R}_{\text {class }}\right)_{\text {new }}$. This is the correction to the fixed term in $s+X Y / 2$ and is the second result.

Using this method the fixed term in GMST was found to be $+14506 \mu$ as, while the fixed term in $s+X Y / 2$ was found to be $+94 \mu$ as.

The fixed term of $+94 \mu$ as in $s+X Y / 2$ corresponds to a value of $-2012 \mu \mathrm{as}$ for the GCRS right ascension of the CEO at epoch (i.e. $+2012 \mu$ as for the constant $C_{0}$ ). We note that the fixed term in GMST compensates both the change in the mean equinox at epoch used as reference for the old and new expressions for GST and the effect in GST of the difference between TT and UT1 at the date of change.

\subsection{Numerical expressions}

The expression for Greenwich Sidereal Time consistent with the IAU 2000A precession-nutation model can be written in the form:

$$
\operatorname{GST00}(\mathrm{UT} 1, t)=\operatorname{GMST00}(\mathrm{UT} 1, t)+E E 00(t),
$$

GMST00 being the polynomial part of GST00 and EE00 its periodic part, with:

$$
\begin{aligned}
& \text { GMST00(UT1, } t)=\theta(\mathrm{UT} 1) \\
& +0 \text { ". } 014506+4612 \text { ". } 15739966 t+1 \text { ". } 39667721 t^{2} \\
& -0{ }^{\prime \prime} .00009344 t^{3}+0 . \prime 00001882 t^{4} \text {. }
\end{aligned}
$$

In order to clarify the link with past expressions for GMST, the following expression provides GMST as an angle, but with $\theta$ expanded as a function of UT1:

$$
\begin{aligned}
& \text { GMST00 }\left(t_{\mathrm{u}}, t\right)(0 \mathrm{~h} \mathrm{UT} 1)=361658^{\prime \prime} 2406561 \\
& \quad+129598159 . .7606402 t_{\mathrm{u}}+4612^{\prime \prime} 15739966 t \\
& \quad+1^{\prime \prime} 39667721 t^{2}-0 . .00009344 t^{3}+0 . .00001882 t^{4}
\end{aligned}
$$

where $t_{\mathrm{u}}$ is the UT1 and $t$ is the TT, both expressed in Julian centuries after $\mathbf{J} 2000$. Note that Appendix B provides the expression in seconds to help the comparison of the $\mathrm{GMST}_{2000}$ and $\mathrm{GMST}_{1982}$ formulas.

The change in the polynomial part of GST from the IAU 1982 model to the IAU 2000A model is, in $\mu$ as and with TAI - UT1 in seconds:

$$
\begin{aligned}
\operatorname{dGMST}(t)= & 14506-274950.12 t \\
& +117.21 t^{2}-0.44 t^{3}+18.82 t^{4} \\
& +47+1.46(\mathrm{TAI}-\mathrm{UT} 1) .
\end{aligned}
$$

The expression for the IAU 2000A equation of the equinoxes is:

$$
\begin{aligned}
\operatorname{EE00}(t)= & \Delta \psi \cos \epsilon_{\mathrm{A}} \\
& +\sum_{k}\left[\left(C_{s, 0}^{\prime}\right)_{k} \sin \alpha_{k}+\left(C_{c, 0}^{\prime}\right)_{k} \cos \alpha_{k}\right] \\
& -0{ }^{\prime \prime} 00000087 t \sin \Omega,
\end{aligned}
$$

the full form of the numerical expression of the second and third lines of (44) being provided in Table 3 and in the electronic table for GST00 as well.

The above expression corresponds to the following change in the equation of the equinoxes, from the IAU 1994 model to the IAU 2000A model, in $\mu$ as:

$$
\begin{aligned}
\mathrm{d} E E(t)= & \sum_{k}\left[\left(C_{s, 0}^{\prime}\right)_{k} \sin \alpha_{k}+\left(C_{c, 0}^{\prime}\right)_{k} \cos \alpha_{k}\right] \\
& -0.87 t \sin \Omega-2640 \sin \Omega-63 \sin 2 \Omega .
\end{aligned}
$$

The definitive expression, in $\mu$ as, for the polynomial part of the quantity $s$ which locates the CEO in a consistent way with the above expressions, is such that, with an accuracy of one $\mu$ as after one century:

$$
\begin{aligned}
(s+X Y / 2)_{\text {polynomial }}= & +94+3808 t-120 t^{2} \\
& -72574 t^{3}+28 t^{4}+15 t^{5} .
\end{aligned}
$$

The full form of the numerical expression for $s$ is available in electronic form at the CDS via anonymous ftp to cdsarc.u-strasbg. fr (130.79.128.5), or from http://cdsweb.u-strasbg.fr/cgi-bin/qcat?J/A+A/406/1135 or http://maia.usno.navy.mil/ch5tables.html, where the full expression for GST00 is also available.

\section{Practical implementation of the new definition of UT1}

There are several ways to implement the IAU 2000 system, and the numerical expressions to be used should be consistent with the procedure required. Using the new paradigm, the complete IAU 2000 procedure to transform from the GCRS to the ITRS is based on (i) the expression (1) for the ERA, (ii) the IAU 2000 expressions for the position $(X, Y)$ of the CIP in the GCRS 
(Capitaine et al. 2003), and (iii) the expressions for the position of the CEO in the GCRS, developed in the paper quoted above and based on the constant and secular terms of its polynomial part, fitted in this paper for ensuring consistency in UT1.

Using the classical paradigm, the recommended option for implementing the new models is to follow the rigorous procedure (Wallace 2002) described by expression (30), composed of the classical nutation matrix using the MHB 2000 series, the precession matrix including four rotations (for $\epsilon_{\mathrm{A}}, \psi_{\mathrm{A}}, \omega_{\mathrm{A}}, \chi_{\mathrm{A}}$ ), using the updated developments for these quantities and a separate rotation matrix for the frame bias. This procedure has to be associated with the use of expressions (41) for GMST and (44) for the equation of the equinoxes, involving the complete form for the expression of the complementary terms.

In the case where one follows the current VLBI procedure and forms the precession-nutation matrix by applying the "total nutations" to the IAU 1976 precession, there are three differences with respect to the previous option which affect the form of GST expression to be used. The first difference is the effect on the matrix of applying all four nutation components not only the luni-solar and planetary components but also the biases in longitude and obliquity and the correction to the 1976 precession rates in longitude and obliquity - at date, when the last two should in fact be applied at epoch. The second is the use of this "total" $\Delta \psi$ in the classical part of the equation of the equinoxes. The third is that the equinox offset is not taken into account in the precession-nutation matrix.

In this case, the new expression, GMST00 $0_{\text {mod }}$ to be used for GMST, together with the EE00 expression for the equation of the equinoxes, has to be derived from expression (41). This is obtained by subtracting (i) the contribution of the equinox offset, (ii) the contribution of the correction to the precession rate in longitude, which is already taken into account in the GMST00 expression and (iii) the cross term between precession and the offset in obliquity. The resulting expression, in $\mu$ as, is:

GMST00 $_{\text {mod }}=$ GMST00 $-14600+274990 t$.

Note that if the quantity $s^{\prime}$ is neglected when allowing for polar motion, the secular term becomes $+274943 t$.

\section{Concluding remarks}

The implementation of the IAU 2000 resolutions, beginning on 1 January 2003, requires the adoption of the IAU 2000 model for the motion of the Celestial Intermediate Pole (CIP) in the Geocentric Celestial Reference System and the adoption of a new definition of UT1. This defines UT1 as linearly proportional to the Earth Rotation Angle (ERA) through a defining relation.

In this paper we have provided expressions to be used to implement the IAU 2000 definition of UT1, using the IAU 2000 precession-nutation model, either in the new (CEO-based) or classical (equinox-based) transformations between the ITRS and GCRS. The computations have been performed so that there is equivalence of the classical and new transformations relative to the rotation about the axis of the CIP when these expressions are used. The new expression provided for GST is in agreement at the microarcsecond level, for one century, with the IAU 2000 expressions for the ERA and for the quantity $s$.

The conventional relationship between ERA and UT1 is theoretically such that it would ensure continuity in phase and rate of UT1 with the value obtained by the previous conventional relationship between GMST and UT1. However, the conditions to be fulfilled in the implementations of the IAU 2000 Resolutions are to ensure continuity in UT1, and as far as possible in UT1 rate, on 1 January 2003, when changing from the current procedure for estimating UT1 from VLBI observations to the new IAU 2000 system, in which $\theta$ (UT1) is a conventional relationship. The determination of the IAU 2000 numerical expressions, linking GST and ERA and locating the CEO, have been performed so that there is no discontinuity in UT1 on 1 January 2003 when changing from the current VLBI procedure to the new one, following equivalent options that are described in the paper. The expected discontinuity in UT1 rate, shown to be unavoidable due to the improved models and the fixed relationship between ERA and UT1, will have an effect on the determination of UT1 less than a few hundreds of microarcseconds over the next century; the corresponding rate variations may reach $5 \times 10^{-15}$.

The resulting expressions have been included in the IERS Conventions 2000.

Note that the formula for GMST is specifically for use with the IAU 2000 precession-nutation model and will need revision on each occasion a new precession model is introduced.

\section{Appendix A: Offsets at J2000}

The transformation between the GCRS and the ITRS requires the use of the frame bias of the mean celestial equatorial frame at J2000 in the GCRS, namely (i) the celestial pole offsets of the mean CIP at J2000, provided by the precession-nutation model with respect to the pole of the GCRS and (ii) the GCRS right ascension of the equinox provided by the precession-nutation model at J2000 (i.e. the equinox offset). This frame bias cannot be computed from theory, but has to be provided by a fit of the precession-nutation model to observations. The IAU 2000 precession-nutation model is associated with the CIP offsets at J2000 with respect to the GCRS pole, which have been derived from VLBI observations (Herring et al. 2002). These offsets have been used in the computation of the expressions for the $X, Y$ coordinates of the CIP in the GCRS consistent with the IAU 2000 precession-nutation model.

The equinox offset, $\mathrm{d} \alpha_{0}$, in contrast, cannot be derived directly from VLBI observations, which are insensitive, to first order, to the position of the ecliptic. The determination of this offset thus requires the use of observations which are dependent on the position of the ecliptic. Consequently, the current VLBI procedure actually ignores the equinox offset, introducing in this way a small spurious distortion in the realization of the CIP of the order of $-\mathrm{d} \alpha_{0} Y$ and $+\mathrm{d} \alpha_{0} X$ (Capitaine 1990) in its $X$ and $Y$ coordinates respectively (i.e. obliquity and longitude $\times \sin \epsilon_{0}$ ).

In our computations, we have tried to take into account the equinox offset in the proper way, which means the value (i) which corresponds to an ecliptic dynamically 
consistent with the IAU's adopted precession-nutation model, (ii) which can be provided by observations with a good accuracy.

The numerical value that we have used is the GCRS right ascension of the mean dynamical equinox at J2000 (-14.6 \pm 0.5 mas) as provided by Chapront et al. (2002) from a fit to LLR observations based jointly on the use of a dynamical theory for the Moon and of VLBI Earth Orientation parameters. The mean equinox of epoch derived in this way corresponds to the definition of the ecliptic in its "inertial" sense to which the recent analytical solutions for the Moon and planets refer. It differs by 93.66 mas from the "rotational dynamical mean equinox of J2000.0" (Standish 1981) as used in the past when referring to the FK5 equinox or to the origin of the JPL ephemerides DE200, and whose the ICRS position has been provided with an uncertainty of 10 mas by Folkner et al. (1994). Note that the $\mathrm{d} \alpha_{0}$ value cannot in fact be considered to be known with an accuracy better than \pm 3 mas, given the uncertainties in the ICRS position both of the MHB 2000 mean equator and of the mean ecliptic.

The MHB precession and nutation, being derived from the theory of the Earth's rotation for a rigid Earth (Souchay et al. 1999) based on the analytical theories for the planets and the Moon, which refer to the inertial dynamical equinox (Simon et al. 1994), are more likely to refer to this equinox.

\section{Appendix B: Comparison with the 1982 expression for GMST}

The IAU-2000-compatible GMST formula given earlier (Expression (41)) differs from earlier formulations in that it is written with respect to ERA. Furthermore, because GMST is better regarded simply as an angle rather than as a time scale, we have used arcsecond units rather than seconds of time as was formerly the convention. In order to compare the 1982 and 2000 expressions, we can write out the ERA and express the coefficients in seconds of Sidereal Time. In seconds, to a resolution of 0.1 microsec:

$$
\begin{aligned}
\operatorname{GMST00}\left(t_{\mathrm{u}}, t\right)= & \mathrm{UT} 1+24110.5493771 \\
& +8639877.3173760 t_{\mathrm{u}}+307.4771600 t \\
& +0.0931118 t^{2}-0.0000062 t^{3} \\
& +0.0000013 t^{4}
\end{aligned}
$$

where $t_{\mathrm{u}}$ is the UT1 and $t$ is the TT, both expressed in Julian centuries after $\mathbf{J} 2000$.

This can also be written as:

$$
\begin{aligned}
& \text { GMST00 }\left(t_{\mathrm{u}}, t\right)=\mathrm{UT} 1+24110.5493771 \\
& +8640184.7945360 t_{u}+307.4771600\left(t-t_{u}\right) \\
& +0.0931118 t^{2}-0.0000062 t^{3}+0.0000013 t^{4}
\end{aligned}
$$

Compare this with the IAU 1982 definition:

$$
\begin{aligned}
\operatorname{GMST} 82\left(t_{\mathrm{u}}, t\right)= & \mathrm{UT} 1+24110.54841 \\
& +8640184.812866 t_{u} \\
& +0.093104 t^{2}-0.0000062 t^{3} .
\end{aligned}
$$

(Note that the conventional formulas for GMST are said to provide the GMST at $0^{\mathrm{h}} \mathrm{UT}$, requiring $t_{u}$ to be in whole days. Such expressions are in fact more general than this and give the difference between GMST and the UT, the steady 4 min per day drawing-ahead of ST with respect to UT; when whole days are ignored the expression happens to equal the GMST at $0^{\mathrm{h}} \mathrm{UT}$ each day. For simplicity, we have generalized the conventional formula by adding to it the UT1, eliminating the apparent restriction to $0^{\mathrm{h}}$ UT. However, it should be borne in mind that accurate computation of these expressions requires careful management of the integer and fractional components if rounding errors are to be kept under control, and it is this practical consideration that lies behind the conventional $0^{\mathrm{h}} \mathrm{UT}$ formulation.)

\section{References}

Aoki, S., Guinot, B., Kaplan, G. H., et al. 1982, A\&A, 105, 359

Aoki, S., \& Kinoshita, H. 1983, Celest. Mech., 29, 335

Capitaine, N. 1990, Celest. Mech. Dyn. Astr., 48, 127

Capitaine, N., \& Gontier, A.-M. 1993, A\&A, 275, 645

Capitaine, N., Chapront, J., Lambert, S., \& Wallace, P. 2003, A\&A, 400,1145

Capitaine, N., Guinot, B., \& Souchay, J. 1986, Celest. Mech., 39, 283

Capitaine, N., Guinot, B., \& McCarthy, D. D. 2000, A\&A, 355, 398

Chapront, J., Chapront-Touzé, M., \& Francou, G. 2002, A\&A, 387, 700

Gontier, A. M. 2002, in IERS Technical Note No. 29, ed. N. Capitaine, et al., Verlag des Bundesamts für Kartographie und Geodäsie, Frankfurt am main, 71

Guinot, B. 1979, in Time and the Earth's Rotation, ed. D. D. McCarthy, \& J. D. Pilkington (D. Reidel Publishing Company), 7

Herring, T. A., Mathews, P. M., \& Buffett, B. A. 2002, J. Geophys. Res., 107, B4, 10.1029/2001JB000390

IERS Conventions 2000, http://maia.usno.navy.mil/ conv2000.html/icc5.ps, draft

Lambert, S., \& Bizouard, C. 2002, A\&A, 394, 317

Lieske, J. H., Lederle, T., Fricke, W., \& Morando, B. 1977, A\&A, 58, 1

Mathews, P. M., Herring, T. A., \& Buffett, B. A. 2002, J. Geophys. Res., 107, B4, 10.1029/2001JB000390

McCarthy, D. D. 1996, IERS Conventions, IERS Technical Note, 21

Simon, J. L., Bretagnon, P., Chapront, J., et al. 1994, A\&A, 282, 663

Souchay, J., Loysel, B., Kinoshita, H., \& Folgueira, M. 1999, A\&A Supp. Ser., 135, 111

Standish, E. M. 1981, A\&A, 101, L17

Wallace, P. T. 2002, in IERS Technical Note No. 29, ed. N. Capitaine, et al., Verlag des Bundesamts für Kartographie und Geodäsie, Frankfurt am main, 65

Williams, J. G. 1994, AJ, 108, 711 